\title{
LA PRESENCIA DE ÁMBAR Y SUB-ÁMBAR (COPAL) EN COSTA RICA: EL CASO DE SAN CRISTÓBAL DE SAVEGRE
}

\author{
THE OCURRENCE OF AMBER AND SUB-AMBER (COPAL) IN COSTA RICA: \\ THE CASE OF SAN CRISTÓBAL DE SAVEGRE
}

\author{
Guillermo E. Alvarado ${ }^{1,2 *}$, Jorge Brenes ${ }^{1,3} \&$ José Brenes $^{4}$ \\ ${ }^{1}$ Instituto Costarricense de Electricidad, apdo. 10032, 1000 San José, Costa Rica \\ ${ }^{2}$ Centro de Investigaciones Geológicas, apdo. 35-2060, \\ Universidad de Costa Rica, San José, Costa Rica \\ ${ }^{3}$ Escuela de Física, Universidad de Costa Rica \\ ${ }^{4}$ Red Ciudadana de Estaciones Meteorológicas, apdo. 290-3015, \\ San Rafael de Heredia, Costa Rica \\ *Autor para contacto: galvaradoi@ice.go.cr
}

(Recibido: 10/08/2012 ; aceptado: 26/11/2013)

\begin{abstract}
In Costa Rica, small fragments of amber are found in Miocene, shallow marine clastic sedimentary rocks of Punta Judas Formation (Middle Miocene), Santa Teresa Formation (Lower Miocene) and Curré Formation (Middle-Upper Miocene). Sub-amber or copal is found relative in abundance in some localities on the ground, in the residual earth soil, in the mountain hills near San Cristóbal de Savegre (Aguirre, Puntarenas). The copal samples have sub-rounded (21.3\%), tabular (29.6\%) and trunk forms (30.4\%), but some have undefined or transitional (18.7\%) morphologies. The color varied from orange/reddish (57.4\%), yellow (31.0\%) and light yellow (11.6\%); and some samples have enclosed insects. The age of the copal is about $1670-1780$ A.D. $\left({ }^{14} \mathrm{C}\right.$ calibrated age $)$. Although these copal occurrences appear to have no economical important value, they could be used for local artisans.

Keywords: Central America, Costa Rica, amber, copal.

RESUMEN: En Costa Rica, pequeños fragmentos de ámbar se encuentran en las rocas sedimentarias marinas clásticas del Mioceno, tales como Formación Punta Judas (Mioceno Medio), Formación Santa Teresa (Mioceno Medio) y Formación Curré (Mioceno Medio-Superior). Sub-ámbar o copal se encuentra en relativa abundancia en ciertas localidades en los suelos residuales en las montañas cerca de San Cristóbal de Savegre (Aguirre, Puntarenas). Las muestras de copal tienen formas sub-redondeadas o aperladas $(21,3 \%)$, tabulares o laminares $(29,6 \%)$ y troncoidales $(30,4 \%)$, pero algunas son intermedias o indefinidas $(18,7 \%)$. Su color varía entre anaranjado/rojizo $(57,4 \%)$, seguido por amarillo $(31,0 \%)$ y menos frecuentemente el amarillo claro hasta blancuzco $(11,6 \%)$; algunas muestras poseen insectos incorporados. La edad del copal está en el rango de edad de 1670-1780 A.D., basado en una datación ${ }^{14} \mathrm{C}$ calibrada. Aunque estas muestras de copal parecen no tener valor comercial, si podrían ser usados por artesanos locales.

Palabras clave: América Central, Costa Rica, ámbar, copal.
\end{abstract}




\section{INTRODUCCIÓN}

En Costa Rica, desde hace casi dos décadas se había mencionado la existencia de ámbares en algunas rocas sedimentarias del Cenozoico (Fig. 1), particularmente en depósitos de areniscas de ambiente deltaico del Mioceno Medio basal en Punta Judas (Seyfried et al., 1994) y aquellos cercanos a los de playa Blanca por Punta Leona, o bien los localizados en Punta Barrigona por Mal País, igualmente asociados con sedimentos del Mioceno Inferior de la Formación Santa Teresa, y al parecer algunos hallazgos en Punta Burica (C. Laurito, E. Gazel, T. Aguilar y P. Denyer, com. escrita, 2012). Más recientemente, durante la excavación del túnel en la ventana de la falla Chánguena (estación $0+452,3 \mathrm{~m}$, a una profundidad o cobertura de 120,4 m; 376,1 m s.n.m. coordenadas CRTM05 $561175 \mathrm{E}-1000190 \mathrm{~N})$, se encontró un fragmento de ámbar contenido en la Formación Curré del Mioceno Medio a Superior (C. Delgado y K. Pérez, com. escrita, 2013).

Los hallazgos más cercanos en América Central, de similar edad, corresponden con los guijarros de ámbar en un nivel conglomerádico rico en moluscos, de 0,5 - $1 \mathrm{~m}$ de espesor, asociado con los sedimentos clásticos de la Formación El Fraile del Mioceno Medio, que quizás alcancen incluso el Plioceno Inferior, aflorantes en el pacífico de Nicaragua (Kolb \& Schmidt, 1991).

En junio de 2012, una cuadrilla de trabajadores del Instituto Costarricense de Electricidad, quienes laboraban en el Proyecto Hidroeléctrico Savegre, particularmente Greivin Granados Elizondo, reconoció en el suelo la presencia de ciertas piezas, que a su criterio, correspondían con ámbar. Dichas muestras fueron analizadas preliminarmente, confirmándose que se trataba de una resina natural. Sin embargo, surgían varias preguntas que debían de evacuarse: a) ¿se trataría de una resina por árboles traídos a la zona en tiempos recientes? b) ¿correspondía efectivamente con una resina fósil, pero no muy antigua (= copal)?, c) ¿correspondía con un verdadero ámbar de edad Paleocena-Miocena, acorde con las rocas detríticas marinas aflorantes en la región, y quizás la fuente de origen de los ámbares previamente

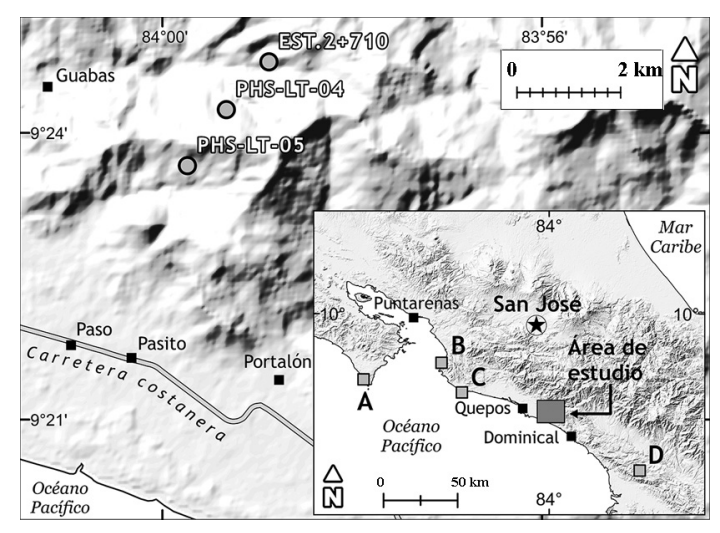

Fig. 1. Mapa de afloramientos de ámbar comprobados: A: Mal País, B: Punta Leona, C: Punta Judas, y D: Changuena. El mapa inserto presenta el detalle de las tres localidades con copal.

mencionados en otras aéreas de país? d) ¿tendría eventuales implicaciones económicas para una artesanía local?

Dada la importancia dentro de la historia geológica costarricense y sus implicaciones paleobotánicas, y eventualmente paleogeográficas y sedimentológicas, así como por ser un sitio potencial a protegerse o en su defecto a ser explotado en forma artesanal por los lugareños, se procedió a realizar una gira de campo en julio de 2012.

\section{CARACTERÍSTICAS DEL ÁMBAR Y DEL COPAL, SU HISTORIA Y DISTRIBUCIÓN MUNDIAL}

A continuación, se aportan algunas definiciones, características, sinonimias, variaciones y usos a lo largo del tiempo, que se le ha dado al ámbar y sus variedades, basado en los trabajos de Schumann (1976), Schlee \& Glöckner (1978), Hunger (1979), Schlee (1980), Poinar (1992) y Schlee et al. (1998).

Definición: El ámbar es una piedra semipreciosa (gema orgánica opaca) hecha de una resina de árbol endurecida y fosilizada, un vidrio polimérico amorfo, que se encuentra en masas redondeadas, a veces laminares, de tamaño discreto, por lo general inferior a $10 \mathrm{~cm}$ de diámetro. El copal es una resina del mismo origen pero varía entre fósil (sub-ámbar fósil), semifósil a actual. Ninguno clasifica como mineral. 
Origen del nombre: En la Grecia Antigua era conocido como elektron o electrum debido a la propiedad que tiene de cargarse negativamente al ser frotado y atraer pequeños trozos de papel o paja. También ha sido llamado electrum, glesum o succinum por los romanos (literalmente "piedra de savia”), y de allí deriva el nombre del ácido que puede estar presente en ciertos ámbares, el ácido succínico. En Prusia se le conocía como Gless, mientras que en alemán originalmente como Bornstein, que luego se cambió a Bernstein; stein significa piedra, bern derivado del alemán bajo y transformado en bernen, que significa quemar ("piedra que se quema"), dado que ya era utilizado para quemar por su agradable olor resinoso. Los amerindios mexicanos utilizaban las palabras apororonalli y xochipalitzli.

Etimológicamente, ámbar proviene del árabe anbar, amber o ambare, que significa, "lo que flota en el mar", y de allí se deriva karabe en persa, ambra en italiano, ambre en francés, amber en inglés y, en nuestro caso, ámbar en español.

Copal viene del náhuatl "copalli" o incienso, dado que se utilizaba como tal y en medicina desde antes de la Conquita.

Sinónimos según las localidades: Succinita (región báltica), gedanita (Polonia), rumanita (Rumanía), simetita (Sicilia), chemawinita o cedarita (Canadá), retinita (México, República Dominicana, Armenia), birmita (Birmania), burmita (Myanmar). Otros ámbares adquieren el nombre compuesto con el lugar de origen, como por ejemplo, ámbar dominicano, ámbar español, ámbar mexicano, etc.

Composición: Se compone de terpenos, alcoholes y éteres, aproximadamente $\mathrm{C}_{10} \mathrm{H}_{16} \mathrm{O}$. La resina se endurece primero por la pérdida gradual de sus componentes volátiles, tales como alcohol y grasa, sufriendo un proceso de polimerización para convertirse en copal, el cual posteriormente pierde la mayor parte de estos componentes durante su enterramiento hasta convertirse en ámbar. Este proceso requiere de al menos 1 a $4 \mathrm{Ma}$ (millones de años), e incluso hasta $17 \mathrm{Ma}$. Los ámbares del báltico contienen ácido commúnico y particularmente el codiciado ácido succínico, este último en diferentes proporciones, procedente de coníferas burseráceas: succino (3 - 8\%) y la burnita $(2 \%)$.
Otros ámbares no poseen este ácido, como el caso de aquellos originados por las Fabáceas, a veces llamados retinita, birnita o tasmanita, entre otros. Por ello, para algunos, estos no deberían de ser considerados como ámbares sensu stricto, pero este criterio no es compartido por todos los autores. Los ámbares pueden contener calcita en las fisuras (burmita) y sulfuros (rumanita, simelita).

Características: El ámbar se encuentra en varios colores, desde amarillo claro y amarillo (los más comunes), marrón claro o naranja, rojizo a rojo profundo, blanco, incoloro, café, y aún azul y verde ahumado o negro. Su color depende del árbol productor de la resina, de la presencia de ciertos minerales y de la alteración de sustancias orgánicas por oxidaciones. Presenta un índice de refracción de 1,540 - 1,6. La transparencia depende de la mayor o menor cantidad de burbujas microscópicas. Es fluorescente, flota en agua de mar, debido a su baja densidad o peso específico: 1,03 - 1,10. En la escala de Mohs 2,0 - 3,0. Su punto de fusión es a $200-380{ }^{\circ} \mathrm{C}$ con un humo negro. Su raya es blanca, brillo resinoso, isótropo, cristalización amorfa, transparente a opaco, con fractura concoidea y sin exfoliación.

El copal es una resina del mismo origen que el ámbar pero varía entre semi-fósil a actual, por lo general del Cuaternario, aunque para otros autores puede ser algo más antiguo. Sus características no difieren mucho, excepto por su particular mayor susceptibilidad a la disolución en disolventes orgánicos, tales como el éter, acetona o cloroformo, que lo vuelve pegajoso. Muchos presentan una dureza en la escala de Mohs de 1 - 3 su punto de fusión es por debajo de $150{ }^{\circ} \mathrm{C}$ y su humo es blancuzco. No todos flotan en agua salada. Bajo la luz ultravioleta se ve más blanca que la del color ámbar, que suele presentar tonos azulados, amarillentos, verdosos y anaranjados, pero pocas veces blancuzcos.

Origen: La resina vegetal proviene principalmente de restos de coníferas, angiospermas, fabáceas y burseráceas. Los árboles emiten un tipo de sabia resinosa como una protección contra las enfermedades y animales herbívoros cuando su corteza es herida debido a una rotura o a un ataque por escarabajos de madera u otros insectos. En 
general, fueron y lo son aún, principalmente los árboles de las familias Araucariaceae (araucarias) y Fabaceae (leguminosas) las que dieron origen a estas resinas fósil, con sus diferentes especies. El ámbar de Europa se formó a partir de la resina del Pinus succinifera, en tanto que en Rumania y en América proviene de farbáceas, que en el caso particular de República Dominicana corresponde con una especie extinta de árbol tropical de hojas anchas, el Hymenaea protera. Su pariente más cercano se encuentra en oriente de África (Hymenaea verrucosa), mientras que en el Caribe, América Central y Suramérica, crecen todavía otros parientes llamado algarrobo, jatoba, curbaril (Brazilian cherry) o el guapinol (Hymenaea courbaril). Los ámbares de Myanmar y Sicilia proceden de burseráceas, tal como el Sequoxylon gypsaceum.

El nombre copal se da en Mesoamérica a especies de las familias Bursera, Protium y Simarubáceas, así como a plantas de otras familias que exudan resinas aromáticas, por lo que se le asocia a varios tipos de árboles en función del país; por ello claramente corresponde a diferentes especies (ver Holdridge \& Poveda, 1975; Gómez, 1986).

Edad: Las muestras de Jordania son del Jurásico. En España, Austria, oeste de Francia, Hungría, Gran Bretaña, Canadá, EE.UU., Líbano, Jordania, Japón, Israel y Siberia, la mayoría son del Cretácico. En el Báltico y China son del Eoceno. En México del Oligoceno al Mioceno. En Rumanía del Paleoceno Superior-Oligoceno. En República Dominicana, Nicaragua, Alemania, Italia, Rusia, Austria, Japón, Malasia y Borneoson del Mioceno. Mientras que en Tanzania son del Mioceno al Pleistoceno, y en Colombia y Japón el copal es del Plioceno-Holoceno.

Preservante fósil: El ámbar es conocido por preservar animales y partes de plantas (p.e. mosquitos, moscas, arañas, hormigas y sus huevos y larvas emergentes, ranas, troncos, hojas, etc.), dado que la composición química de la resina actúa como desecante y como componente antibiótico, quedando atrapados pero sin encogimiento.

Yacimientos: Aunque se le ha encontrado en muchos lugares, solo hay una veintena de depósitos sobresalientes: Europa Oriental (Báltico) ta- les como Dinamarca, Suecia, norte de Alemania, Polonia Lituania, Letonia, Rusia y Myanmar (antigua Birmania); en Latinoamérica en México y República Dominicana. Otros lugares son España, Canadá, Líbano, Israel, Jordania, oeste de Francia, sur de Gran Bretaña, Francia, EE.UU., Japón, Tanzania, Nicaragua y Colombia.

Arqueo-ocurrencias: El ámbar es una de las piedras más antiguas llevadas como joya en el cuerpo. La pieza más antigua trabajada por el hombre data de hace unos 30000 años, y se encontró en Hannover, Alemania, o como collares en el norte de Europa de hace 8000 años a.C. Se le encontró también en las ruinas atribuidas a Troya, así como en las tumbas micénicas. Los fenicios intercambiaban el ámbar u "oro del báltico" en los pueblos bálticos antiguos desde aproximadamente 3000 años a.C., en la Europa Meridional, a través de rutas de comercio o "ruta del ámbar" que llegaban incluso al lejano oriente. Desde 1283, los caballeros teutónicos, después de regresar de las cruzadas, se convertirían en gobernadores de Prusia y de las fuentes bálticas de ámbar, así como de la manufactura, castigando transgresores con la muerte en la horca. Posteriormente, por unas cinco centurias, el ámbar fue usado con fines religiosos en la confección de sartas de cuentas de rosarios.

En América, se le conoce su uso por lo menos desde el 700 a.C. por los Olmecas y posteriormente los mayas, como una resina aromática y como ornamentación. Se tiene igualmente su mención de cuando Colón llegó en 1492 a la isla La Hispañola (hoy República Dominicana). En Costa Rica hasta el momento no se tiene registros de ámbar o copal en los hallazgos arqueológicos.

Propiedades místicas y curativas atribuidas: En la antigüedad, la mitología griega consideraba que el ámbar eran lágrimas solidificadas de las Helíades, que brotaban por la muerte de su hermano Faëton, ahogado al caerse con del carro del Sol. Por otra parte, Sófocles narra la leyenda de Meleagrides (Meleager), cuyas hijas fueron transformadas en aves, y una vez al año hacían un vuelo de Grecia a la India, y las lágrimas que dejaban caer, se convertían en gotas de ámbar. El jardín de las hijas por la noche, las Hespérides, poseía el secreto de la inmortalidad, guardado en 
los manzanos que producían frutos dorados (ámbar ?), cuidado por un dragón de siete cabezas. Cuando Atlas mató al dragón, obtuvo los frutos y se hizo inmortal. Por ello, los polvos de esta resina, llamados de ambrosía (ambrotos, del griego, mortal), eran utilizados para obtener la salud y la inmortalidad. De ahí su uso extendido en la medicina. Hércules, el héroe más popular de la mitología griega, tenía un escudo con ámbar, que reflejaba rayos segadores sobre sus enemigos.

En el Éxodo (30: 34-38) se menciona la quema de copal para hacer más propicio el espacio sagrado: "Dijo además Jehová a Moisés: Toma especias aromáticas,... y lo pondrás delante del testimonio en el tabernáculo de reunión, donde yo me mostraré a tí. ...Es un jugo de color ámbar que se desprende de los tallos de un arbusto ...".

Ya al inicio de nuestra era, Popea, la esposa de Nerón, con su cabellera color ámbar, convirtió dicha piedra en la gema más deseada y usada en Europa. Sin embargo, el primero en presentar evidencia de que era en realidad sabia de árboles similares al pino, y que su origen provenía del norte y que con frecuencia contenía insectos, fue Plinio el Viejo, en el siglo I de nuestra era.

De igual modo, se pensaba que poseía propiedades místicas o mágicas, siendo utilizado como talismán o remedio medicinal o ingrediente en las medicinas, particularmente un elixir antibiótico. Por no ser un cristal, es cálido al tacto, y por ello se le atribuía el poder del dios Sol, utilizándosele en ceremonias del fuego en todo el mundo. Los chinos creían que el ámbar contenía en su interior el alma del tigre, la que después de su muerte, se escondía en la tierra; aún hoy día, el ideograma en el alfabeto chino para denominar el ámbar evoca la imagen del tigre. Lo mismo se aplica a la caligrafía japonesa, adaptada del chino.

Aún se le usa para proteger a los niños contra el "mal de ojo", o para supuestamente influir positivamente sobre el sistema endocrino, el bazo y el corazón, para purificar el cuerpo, la mente y el espíritu, y para limpiar habitaciones de la negatividad acumulada.

Solo el ámbar báltico posee ácido succínico, utilizado todavía hoy en la medicina y la industria farmacéutica para la cura de diferentes dolencias.

\section{COPAL EN COSTA RICA: EL CASO DE SAN CRISTÓBAL, AGUIRRE}

\section{Metodología}

Se procedió a realizar una gira al campo para reconocer las localidades y ver el contexto geológico de los sitios y sus alrededores. Se observaron los cortes naturales y artificiales, así como las perforaciones a núcleo continuo. Se consultó con las personas que lo localizaron y se tomaron muestras in situ. Posteriormente, de las muestras recolectadas, se estudiaron 263 muestras para un total de $888,65 \mathrm{~g}$, que se describieron macroscópicamente y se realizaron mediciones morfométricas con un vernier con una precisión de 1/20. Sin embargo, hay que tomar en consideración, que la masa total varía en algunos gramos en cada análisis, dado que se iba perdiendo material bajo la forma de esquirlas, debido a lo frágil que resultan algunas muestras. Sin embargo, para los resultados, resulta despreciable dichas pérdidas. Para determinar la abundancia relativa de las geoformas y de colores de las piezas, se procedió a agruparlas, pesarlas en una balanza de precisión (tipo BX 620S, Shimadzu Co., con una precisión de $\pm 0,01 \mathrm{~g}$ ) y a calcular los porcentajes. Los colores de las piezas fueron determinados con los ejemplares limpios de barro, pero sin pulir. Muchos de ellos, a la hora de pulirlos, suelen adquirir colores más claros y sus translucidez o incluso transparencia, incrementa.

Para probar la flotabilidad en agua salada, se procedió a poner $25 \mathrm{cl}$ de agua con $4 \mathrm{cl}$ de sal, o 10 cucharadas de sal en $250 \mathrm{ml}$ de agua. La densidad se determinó en el laboratorio de Física de la Universidad de Costa Rica, utilizando el principio de Arquímedes. A unas esquirlas se les atacó con éter para ver su grado de reacción, y también se les determinó la densidad. Algunos ejemplares fueron pulidos con papel de lija de diferente número para poder fotografiar algunos de los insectos con el microscopio petrográfico y binocular. Al no ser las piezas planas y con el fin de no dañar muchos de los ejemplares, no se pudieron fotografiar muchos de los ejemplares de insectos hallados. 
Se mandó a datar una muestra con radiocarbono al laboratorio Beta Analytic Inc., (número de laboratorio Beta-331104). El laboratorio presentó una serie de calibraciones basado en el trabajo de Reimer et al. (2009).

\section{Localidad}

Las muestras se recolectaron en las serranías cerca del poblado de San Cristóbal de Savegre, cantón Aguirre, provincia de Puntarenas. En tres sitios se informó la presencia de piezas "ambarinas" (Fig. 1). La primera fue en la estación topográfica con fines de estudios geofísicos en la línea de conducción, la estación $2+710$ cuyas coordenadas (CRTM05) son $1040747,657 \mathrm{~N}$ - 502054,990E (Lambert Sur, 373,751N - 465,232E, Hoja Savegre), con una elevación de 393 m.s.n.m.; el segundo sitio se ubica en la perforación 4 (PHSLT-04), en el estacionamiento 3+ 940 cuyas coordenadas son $1039834,423 \mathrm{~N}$ - 501234,016E (Lambert Sur, 372,797N - 464,465E, Hoja Savegre), con una elevación de 375 m.s.n.m.; y el tercer sitio se localiza en la perforación 5 (PHSLT-05), en el estacionamiento 5+230, coordenadas 1038753,566N - 500483,016E (Lambert Sur, $371,788 \mathrm{~N}$ - 463,661E, hoja Savegre), con una elevación de 380 m.s.n.m. Dichos sitios se encuentran separados por una distancia de $2,5 \mathrm{~km}$ en línea recta, y entre ellos, al parecer no se ha localizado muestras de resina fósil. La topografía es abrupta con fuertes pendientes de más de $30^{\circ}$. Las piezas de resina suelen encontrarse en tramos lineales relativamente restringidos de escasos 30 $\mathrm{m}$ en el caso de la estación y de unos $50 \mathrm{~m}$ en el caso de las perforaciones. Sin embargo, la abundancia relativa es significativamente mayor en la estación $2+710$ con respecto a la perforación 4 y trazas en la 5.

Las piezas suelen encontrarse superficialmente, en las primeras decenas de centímetros, y en el caso particular de la estación, a no más de $20 \mathrm{~cm}$ como máximo, comprobadamente a $10 \mathrm{~cm}$ dentro del suelo residual y coluvial. No se observaron incrustaciones de piezas ambarinas en las perforaciones, afloramientos de roca frescas ni en las de rocas profundamente meteorizadas. Fuera de dichas localidades, la presencia de piezas es reducida a nula, y en su mayor parte las que se encuentran, o fueron removidas en forma antrópica en el camino que lleva a las perforaciones o por la acción de escorrentía de las aguas superficiales.

\section{Descripción}

Las muestras recolectadas presentan varios colores, desde secciones incoloras dentro de una pieza a amarillo claro y amarillo (los menos comunes), hasta marrón claro o naranja o rojizo, los más comunes. Algunas piezas son semitransparentes, todas traslúcidas, en superficie con pátina blancuzca o grisácea, donde pueden presentar grietas de contracción, aspecto oqueroso o una superficie tosca semilisa, o bien presentar estriaciones, posiblemente en correspondencia con calcos de la superficie de la corteza arbórea original o de algunas ramas. Internamente, presentan un brillo resinoso, vidrioso a nacarado, fractura concoide, con partículas microscópicas de impurezas (polvo, arena, detritos vegetales, insectos u sus huevos) y burbujas. Su color varía entre anaranjado/rojizo $(57,4 \%)$, seguido por amarillo $(31,0 \%)$ y menos frecuentemente el amarillo claro hasta blancuzco (11,6\%) (Fig. 1 y cuadro 2).

Sus formas se pueden agrupar principalmente en cuatro grupos: a) las formas redondeadas o perlas (equivalente a las formar cristalinas coloformes u ovoides), que representan el 21,3\%, b) las alargadas con forma de troncos ("troncoidales"), en un $30,4 \%$, c) las laminares (hojosas o tabulares) con un $29,6 \%$, y d) las formas transicionales, intermedias o indefinidas, en un $18,7 \%$ (Fig. 2 y cuadro 1).

La perla (término usado para resinas arbóreas similares y recientes) más grande mide 3,4 x 1,9 x 1,3 cm, aunque las más esféricas poseen un diámetro máximo de $1,8 \mathrm{~cm}$, siendo su tamaño promedio de 2,0 x 1,5 cm. Las que poseen forma de troncos, tienen un tamaño de $6-8 \times 2,5-3 \times$ $0,5-2,0 \mathrm{~cm}$ (un caso excepcional es de $15 \times 4$ $\mathrm{cm})$. En cambio, las que poseen forma laminar o tabular tienen como tamaño máximo $8,0-7 \mathrm{~cm} \mathrm{x}$ $1,0 \mathrm{~cm}$, y un grosor $2 \mathrm{~mm}$. Es decir, las perlas son menos abundantes $(21,3 \%)$ y suelen tener colores 
Cuadro 1

Cantidad porcentual de las formas predominantes de copal en los dos sitios

$\begin{array}{cccccc}\text { Localidad } & \text { Troncoidales } & \text { Perlas } & \text { Láminas } & \text { Indeterminados } & \text { Masa total } \\ \text { Est. } 2+710 & 29,44 \% & 20,11 \% & 31,50 \% & 18,95 \% & 823,70 \mathrm{~g} \\ \text { Perforación } 4 & 43,15 \% & 36,88 \% & 4,70 \% & 15,27 \% & 63,78 \mathrm{~g} \\ \text { Cantidad de muestras } & 34 & 84 & 60 & 85 & 263 \\ \text { Masa total (g) } & 269,98 & 189,14 & 262,54 & 165,82 & 887,48 \mathrm{~g} \\ \text { Total } & 30,42 \% & 21,31 \% & 29,58 \% & 18,68 \% & 99,99 \%\end{array}$

amarillentos (Cuadros 1 y 2). Sin embargo, a la hora de pulir las piezas, suelen adquirir colores más claros.

En la escala de Mohs su dureza es de aproximadamente 2,5. En luz negra, brilla fosforescentemente y al ser frotada con seda, se vuelve piezoeléctrica. Flota en agua salada saturada (densidad del agua de mar $1,027 \mathrm{~g} / \mathrm{cm}^{3}$ ) pero no en agua dulce, dado que posee una densidad de 1,056 $1,065 \mathrm{~g} / \mathrm{cm}^{3}$ (promedio $1,06 \mathrm{~g} / \mathrm{cm}^{3}$ ). Presenta cierta susceptibilidad a la disolución en disolventes orgánicos, tales como el ser pegajoso en éter.

\section{Bio-inclusiones}

Se llegaron a observar pequeños insectos voladores de tamaño milimétrico, particularmente en las muestras con forma de troncos, no así en las muestras con forma de perlas o láminas, posiblemente por funcionar a modo de tapón obstructor de la herida, y no como un derrame de sabia corriendo por la corteza del árbol, atrapando insectos o siento una trampa para ellos (Fig. 3).

\section{Edad}

La edad de radiocarbono sin calibrar resultó de $130 \pm 30$ años a. P. (el presente es tomado como 1950 A.D.), por lo que la mejor fecha calibrada a nuestro criterio y con base en los resultados del laboratorio sería la del año 1670 a 1780 A.D., es decir en el tiempo de la colonia española.

\section{Origen}

La resina vegetal todavía guarda ciertas formas posiblemente de la corteza del árbol que le dio origen, pero no se pudo determinar su familia. Los árboles de araucarias y pinos, una de las fuentes del ámbar americano, al parecer nunca alcanzaron esta parte de América Central Austral (Gómez, 1986). Las perlas descritas en esta localidad, en cambio, son idénticas a aquellas encontradas en otros lugares de Costa Rica asociadas al guapinol. Dichas perlas, o bolsas de resina cristalizada, se forman en antiguas heridas, que son absorbidas al engrosar el tronco. Así que probablemente pro-

Cuadro 1

Cantidad porcentual de las formas predominantes de copal en los dos sitios

\begin{tabular}{|c|c|c|c|c|c|c|c|}
\hline \multirow{2}{*}{ Forma del ámbar } & \multicolumn{2}{|c|}{ Amarillo claro } & \multicolumn{2}{|c|}{ Amarillo } & \multicolumn{2}{|c|}{ Anaranjado / rojizo } & \multirow{2}{*}{ Masa total (g) } \\
\hline & Masa (g) & $\%$ & Masa (g) & $\%$ & Masa (g) & $\%$ & \\
\hline Perlas & 67,81 & 35,87 & 70,13 & 37,10 & 51,09 & 27,02 & 189,03 \\
\hline Troncoidales & 2,76 & 1,02 & 56,50 & 20,93 & 210,66 & 78,04 & 269,92 \\
\hline Láminas & & & 90,97 & 34,71 & 171,08 & 65,28 & 262,05 \\
\hline Indeterminadas & 32,72 & 19,75 & 57,03 & 34,42 & 75,90 & 45,82 & 165,65 \\
\hline Total & 103,29 & 11,65 & 274,63 & 30,97 & 508,73 & 57,38 & $\begin{array}{l}886,65- \\
888,65\end{array}$ \\
\hline
\end{tabular}




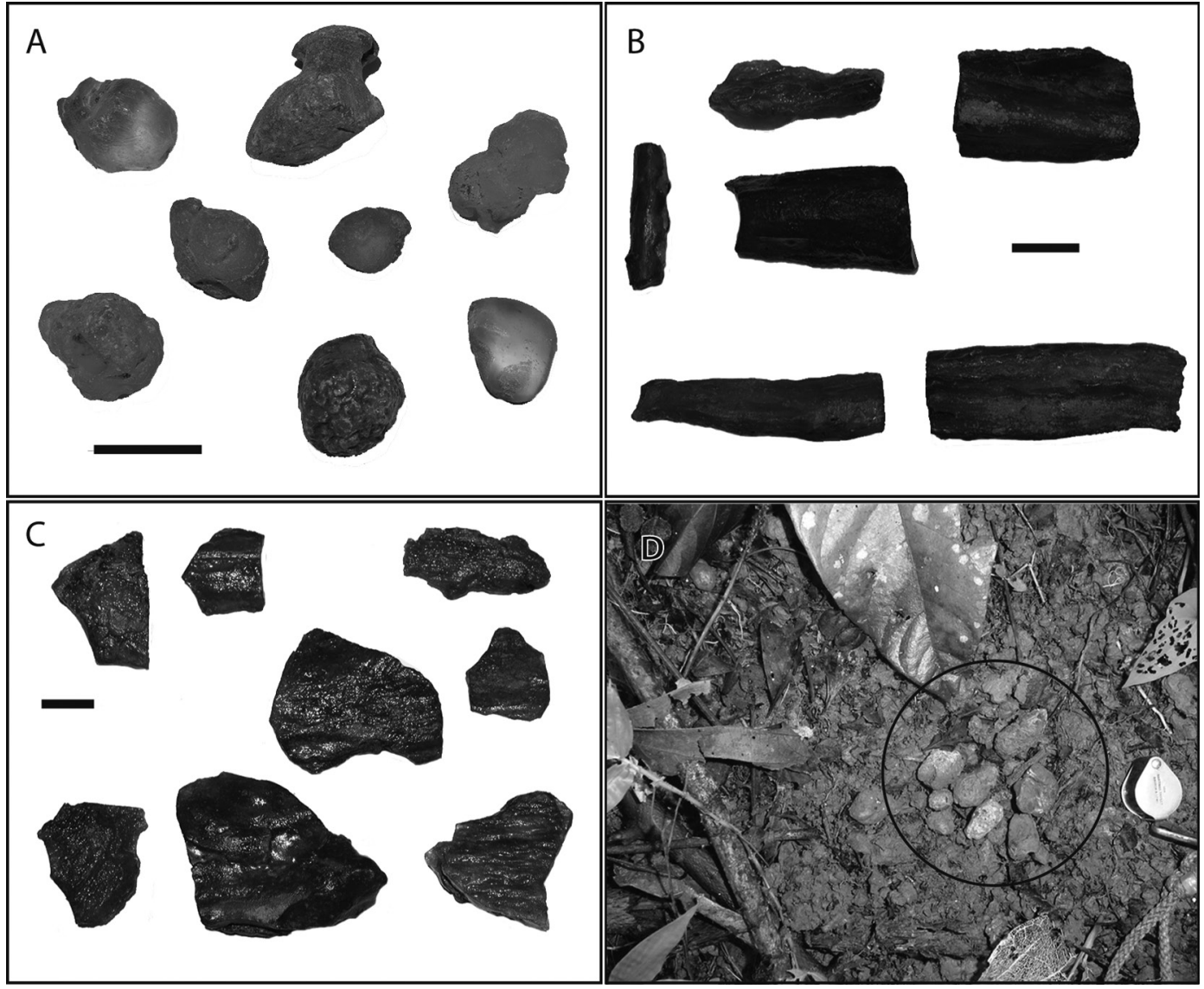

Fig. 2. Ejemplos de las diferentes formas del copal encontradas: a) perlas, b) troncoidales, c) láminas y d) modo como se encuentran en el campo. La barra de la escala representa $2 \mathrm{~cm}$.

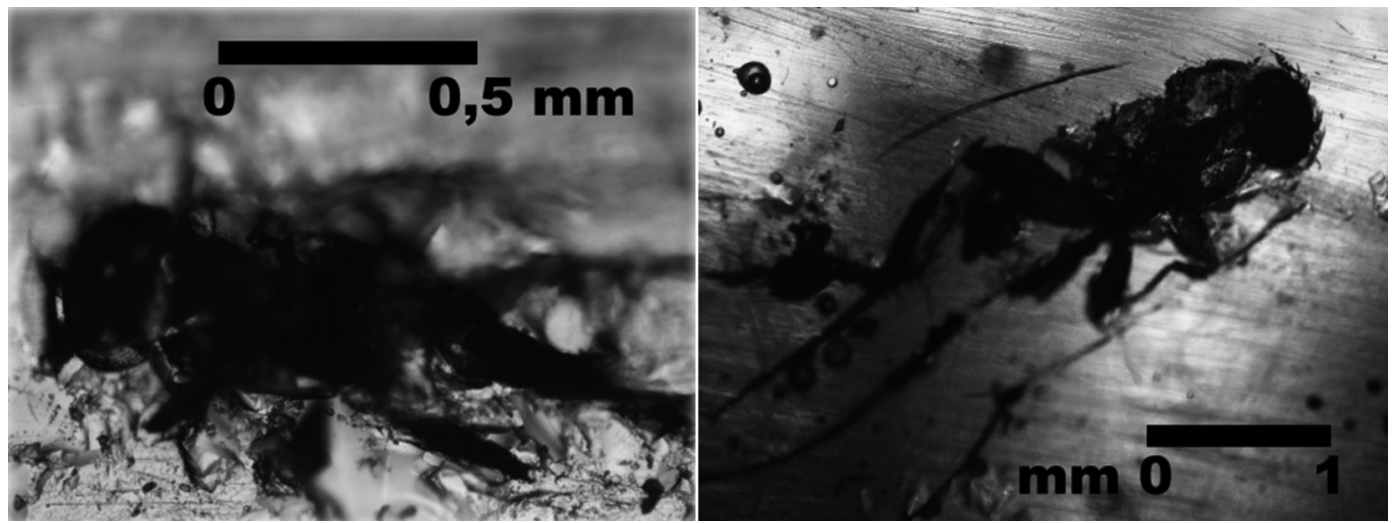

Fig. 3. Ejemplos de insectos atrapados en el copal, vistos bajo el microscopio (Fotografías Manuel Barrantes). 
cedan del guapinol (Hymenaea courbaril), una leguminosa arbórea (Fig. 4). El guapinol actual vive entre el nivel del mar y unos 900 m.s.n.m. (a veces incluso $1300 \mathrm{~m}$ ), dentro del rango de la altura del hallazgo, que suele estar muy restringido al momento, entre unos 375 y 400 m.s.n.m. Otros posibles orígenes podrían ser el árbol de Santa María, también conocido como guarandí o arary (Calophyllum brasiliensis), el bálsamo del Perú (Myroxylom balsamum). Todos ellos se presentan en el área de estudio (ver p.e. Holdridge \& Poveda, 1975; D. Arguello M., com. escrita, 2012). Pese a que con el análisis visual del copal no es posible su identificación, todo parece sugerir al guapinol como el candidato más probable.

\section{CONCLUSIONES}

Un nuevo hallazgo en San Cristóbal de Savegre (Aguirre, Provincia de Puntarenas), permite determinar la presencia de una resina sub-fósil del tipo copal (sub-ámbar), de la época colonial (1670-1780 A.D.), en tres sitios de la localidad, con alturas entre 375 y 400 m s.n.m. El candidato más probable en dar origen a esta resina es el guapinol (Hymenaea courbaril).

Su poca abundancia relativa, su baja calidad y edad, la excluyen de ser un sitio de explotación comercial, aunque no deja de ser de interés científico.

\section{AGRADECIMIENTOS}

A la cuadrilla de Geofísica del ICE, por su colaboración: Alfredo Calderón Navarro, Gerardo Torres Méndez, Greivin Granados Elizondo, Álvaro Sánchez Chaves, Otilio Garia Guzmán, Alejandro Moya Sánchez, Marco Torres Aguilar, Mauricio Ramos Ruiz, Cesar Pérez Zúñiga, Edwin Mora Arias, Enrique Cordero Rojas, Adrián Granados Elizondo, Ángel Monge Gamboa, Jorge Mesén Alemán, Luis Diego Calvo García.

Al colega Salvador Brouwer, hijo del Pompilio Brouwer, conocido como el "Padre de la Industria del Ámbar en la República Dominicana”,

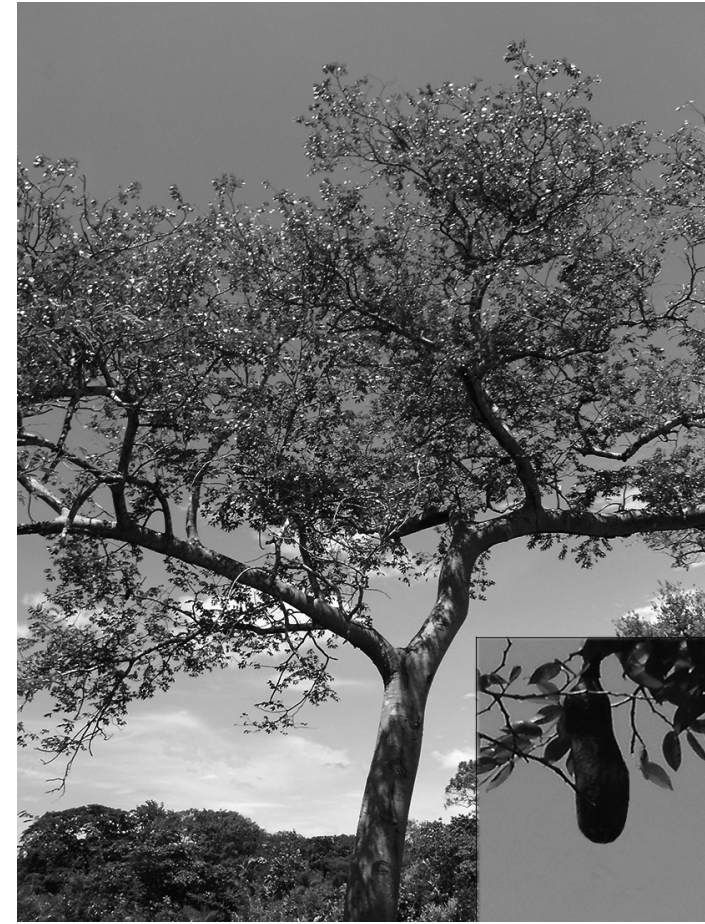

Fig. 4: Árbol de guapinol en Guanacaste y un recuadro con su fruto (aprox. $10 \mathrm{~cm}$ de largo).

por proveer de una nutrida literatura especializada y por sus valiosos comentarios. Los comentarios forestales de Diego Arguello Murillo fueron muy provechosos. Manuel Barrantes fotografió los insectos y Alberto Vargas colaboró con la figura 1. Los señores Luis Madrigal y Francisco Arias, apoyaron con comentarios y documentación acorde con su naturaleza investigativa. Percy Denyer criticó el texto con aportes substanciales.

El análisis de radiocarbono fue realizado gracias al aporte de la Red Ciudadana de Estaciones Meteorológicas (RECIEM).

\section{REFERENCIAS}

GÓMEZ, L.D., 1986: Vegetación de Costa Rica.327 págs. EUNED, San José.

HOLDRIDGE, L.R. \& POVEDA, L.J., 1975: Árboles de Costa Rica.- 546 págs. Centro Científico Tropical, San José. 
HUNGER, R., 1979: The Magic of Amber.131 págs. Chilton Book Co., Randor, Pennsylvania.

KOLB, W. \& SCHMIDT, H., 1991: Depositional sequences associated with equilibrium coastlines in the Neogene of southern Nicaragua.- Spec. Publs. int. Ass. Sediment., 12: 259-272.

POINAR, G.O. Jr., 1992: Life in Amber.- 275 págs. Stanford University Press, Stanford.

REIMER, P.J., BAILLIE, M.G.L., BARD, E., BAYLISS, A., BECK, J.W., BLACKWELL, P.G., BRONK RAMSEY, C., BUCK, C.E., BURR, G.S., EDWARDS, R.L., FRIEDRICH, M., GROOTES, P.M., GUILDERSON, T.P., HAJDAS, I., HEATON, T.J., HOGG, A.G., HUGHEN, K.A., KAISER, K.G., KROMER, B., McCORMAC, F.G., MANNING, S.W., REIMER, R.W., RICHARDS, D.A., SOUTHON, J.R., TALAMO, S., TURNEY, C.S.M., VAN DER PLICHT, J. \& WEYHENMEYER, C.E., 2009: Intcal09 and Marine04 radiocarbon age calibration curves, 0-50,000 years cal BP.Radiocarbon, 51(4): 1111-1150.
SCHLEE, D., 1980: Bernstein-Raritäten. Farben, Structuren, Fossilien, Handwerk.- 88 págs. Staatlichen Museums für Naturkunde in Stuttgart, Múnich.

SCHLEE, D. \& GLÖCKNER, W., 1978: Bernstein.- Stuttgarter Beiträge zur Naturkunde, 8(C): 1-72.

SCHLEE, D., JIMÉNEZ, A., ITURRALDEVINENT, M., VICIOSO, C., CARIDAD, J. \& LANZETTI, G., 1998: Mundo de ámbar. Los secretos del ámbar dominicano.119 págs. Museo Mundo de Ámbar, Santo Domingo.

SCHUMANN, W., 1976: Edelsteine und Schmucksteine.- 255 págs. BLV Verlagsgesellschaft, Múnich. [Trad. español: J. LALUCAT, 1978: Guía de las piedras preciosas y ornamentales.- 148 págs. Ed. Omega, Barcelona].

SEYFIRED, H., KRAWINKEL, H. \& AGUILAR, T., 1994: Significance of stratigraphic bounding surfaces in a shallow marine, sand-swamped forearc basin: a case study from the Punta Judas Formation (Miocene, Costa Rica).- Profil 7: 293-323. 